\title{
FROM THE ARCHIVES
}

\section{JAN PATOČKA (Prag) Das Urchristentum im Rahmen der antiken Religionen ${ }^{1}$}

In dieser kleinen Schrift bietet der Meister der Neutestamentarischen Kritik und Interpretation seinen Lesern einen umfassenden Überblick über die Verortung des Urchristentums auf der geistigen Landkarte der alten Welt. Es ist das Werk von einem der heute herausragenden Forscher, verwurzelt in der Schule der deutschen Theologie und der deutschen philosophischen Strömungen der ersten Hälfte unseres Jahrhunderts. Den Ausgangspunkt seiner Interpretation bilden die allgemeinen Grundhaltungen, in denen sich das Selbstverständnis des antiken Menschen ausdrückt und - insofern diese Haltungen auch uns berühren - das Selbstverständnis des Menschen überhaupt. In fünf Kapiteln analysiert er hier das Alttestamentarische Erbe (die Grundlagen des jüdischen Monotheismus), das Judentum der Zeit Jesu, die griechische Weltanschauung, den Hellenismus und schließlich das Urchristentum (vor allem Paulus und das Johannesevangelium).

Die Achse des Buches bildet, sachlich wie auch methodisch, der Kontrast zwischen dem judaistischen und dem hellenischen Verständnis des Menschen. Deutlich werden die Züge des altjüdischen Theismus herausgearbeitet, sein supranaturaler und voluntativer Charakter, in dem weder der Begriff noch das Problem von Natur und Natürlichkeit vorkommen. Wesentlich ist in der jüdischen Haltung die historische Auffassung der Welt, die Unvermeidlichkeit eines historischen Ausdrucks des Glaubens, der hier die unbedingte Abhängigkeit von der Macht Gottes meint. Die Geschichte selbst wiederum wird darin nicht a tergo, kausal, ausgelegt, sondern a fronte, final, von ihrem Ende und ihrer Erfüllung her, eschatologisch, nicht immanent historisch und politisch wie bei den Griechen. Das symbolisch-sinnliche Modell der Erkenntnis ist nicht das griechische Schauen, sondern das Hören, welches das Wort der Weisung vernimmt. Auch Wahrheit ist ursprünglich Weisung, die Anerkennung verlangt, keine Aussagewahrheit wie bei den klassischen Griechen. Ebenso wenig ist Weisheit theoretisches Wissen, vielmehr Gottesfurcht, zu unterscheiden von der Angst, von der sie befreit und so dem Leben Sicherheit verleiht. Die Gottesfurcht, der Respekt vor der göttlichen Weisung, gibt dem Menschen Sicherheit ganz ohne jeden seichten Optimismus; er weiß um das Leiden, er weiß um das menschliche Unterworfensein durch die Natur, um seine Nichtigkeit vor Gott; gegen das Leiden des Einzelnen hilft keine

\footnotetext{
${ }^{1}$ Rezension zu Rudolf Bultmanns gleichnamigem Buch (Zürich: Artemis Verlag, 1949).
} 
allumfassende Harmonie. Vor Gott soll sich der Mensch nicht fühlen wie das Glied eines autonomen gesellschaftlichen Ganzen oder wie der spezielle Fall einer allgemeinen Regel, sondern er soll hoffen auf die Zukunft und sich dabei der unergründlichen göttlichen Ordnung unterwerfen. Dieses hoffende Warten, bei dem das Schicksal des Einzelnen eingelassen ist in die Schicksale des ganzen Volkes, sich verlassend auf den göttlichen Plan, ist der alt-testamentarische Glaube.

Im Unterschied zu anderen vorderasiatischen Religionen ist Gott hier nicht an einen Ort gebunden, sondern an eine Nation, an ein Volk. Dieses Volk aber ist keine Gemeinschaft Freier und Gleichberechtigter, hervorgebracht durch einen gemeinsamen Willen. Es entsteht vielmehr durch die Treue zur Geschichte und Tradition, d.h. durch Gottesfurcht. Die Feiertage vergegenwärtigen dieses Geschehen, die Geschichte des Bundes mit dem Herrn. Im subjektiven Erleben der Geschichte dieses Bundes begründet sich das Bewusstsein einer Erwählung als Volk, die allerdings scharf zu unterscheiden ist von aller Selbstgerechtigkeit: Gott hat erwählt, Gott kann auch aus freien Stücken verwerfen. Dadurch entsteht (bei den Propheten) die Tendenz zu einer Eschatologisierung der Idee des Bundes. Folglich liegt auch der Gedanke der göttlichen Herrschaft immer im Konflikt mit dem weltlichen Königreich, ebenso wie das Prophetentum von Beginn an in einen Konflikt zu den Tendenzen einer neuen Staatlichkeit und gesellschaftlichen Organisation des Königstums Davids tritt, wodurch aber es aber diese Staatlichkeit überleben und in die Theokratie Ezras übergehen kann.

Anthropologisch betrachtet kennt das Alte Testament nicht den typisch griechischen Dualismus von Körper und Seele. Stattdessen setzt es den Unterschied von Fleisch und Seele als zwei Seiten desselben Lebewesens. Es gibt keine Unsterblichkeit, erst spät dringt aus iranischen Quellen die Idee einer Auferstehung von den Toten ein. Das Sterben ist kein Akt, in dem das Leben harmonisch ausatmet, so wie in der griechischen Weisheitslehre. Es gibt nicht das Problem eines eigentlichen (wahren) Lebens wie bei den Hellenen, somit auch nicht das eines „geistigen“ Lebens: ein langes und glückliches Leben natürlicher Art ist das höchste menschliche Gut. Deshalb müssen hier alle ethisch-erzieherischen Ideen und Ideale, die so typisch für das Griechische sind, fehlen. Keine Mystik, keine Persönlichkeit, natürlich auch keine kulturelle und literarische Tätigkeit, die sich darum drehen würde: So wird z.B. die Dichtung ausschließlich zum Ausdruck einer religiösen Tendenz, zu Lobpreis, Dank, Bitte, Klagelied. Gut und Böse entspringen nicht dem Ideal einer Autonomie der menschlichen Person, gehen nicht aus einer Gestaltung der menschlichen Natürlichkeit hervor, sondern aus der Beziehung zum Gesetz: Gerechtigkeit ist dessen aufrichtige Erfüllung. Die Ethik, formuliert in einer Reihe von Verboten ohne jedes Bemühen um rationale Systematisierung, ist sozial, aber nicht politisch. Die Grenze zwischen kultischen und mo- 
ralischen Vorschriften ist fließend, was bei einigen Propheten zu der Auffassung führt, Gerechtigkeit sei die einzige Forderung der Frömmigkeit. Die Sünde besteht in Selbstverschließung, Eigensinn, Mangel an Vertrauen und Glauben (nicht erst im lästerlichen Hochmut). Der Mensch ist auf die Vergebung Gottes, auf seine Gnade angewiesen, und was zu ihr führt, ist die Anerkennung des göttlichen Urteils, dass wir den Verlust unseres Lebens verdienen: Buße. Die Gnade Gottes wird nicht dem Einzelnen gewährt, sondern dem israelischen Volk durch eschatologische Hoffnung auf göttliche Vergebung am Ende der Zeiten.

Es ist erkennbar, wie Bultmann in seiner Erfassung der typisch alt-testamentarischen Haltung einen gleich großen Abstand zu wahren sucht von der Ableitung der gesamten komplizierten Struktur aus einem einzigen Prinzip (wie das etwa einige Vertreter des deutschen Idealismus versuchten) wie auch von einem Versinken in der Endlosigkeit des Empirismus. Was fing man aber nun an mit diesem Erbe des historischen Judentums beim Kommen Jesu? Das Judentum dieser Zeit schuf die Frömmigkeit der Synagoge, die sich nicht auf das Kultische, sondern auf die Auslegung der Schrift konzentrierte. Dadurch gewann es zwar feste Zuversicht und Bindung an die historische Tradition, erlaubte aber zugleich einen Verfall. Denn die Geschichte, an die sie sich heftete, ist etwas Vergangenes, Totes, keine gegenwärtige, dringende Geschichte. Auch die Auffassung des Herrn und seines Gesetzes wird enthistorisiert. Gott wird zum Richter der ganzen Welt, zum Herrn aller Völker, seine unendliche Distanz bewirkt, dass er von allen gleich weit entfernt ist. Es wurde zwar das gesamte Leben zum Kult, als die großen Feiern ihre außerordentliche Bedeutung verloren und die Synagoge den Tempel ersetzte, aber man erleichterte sich dieses durch eine kodifizierende Formelhaftigkeit. Die Moral ist überwiegend negativ gefasst, und unter einem Übermaß von Reinigungsvorschriften sowie anderer Gebote und Verbote fällt ihr Sinn dem Vergessen anheim. Selbst wenn es nicht an gelegentlicher Reflexion fehlt, was die alles regulierende Einheit dieser Masse an Formeln ist und wo deren moralische Bedeutung liegt, verleiht es dem jüdischen Leben insgesamt doch einen Zug der Kleinlichkeit und Ängstlichkeit, durch den es sich unvorteilhaft von der griechischen eleutheria unterscheidet. Große und kleine Forderungen oder Vorschriften stehen unterschiedslos nebeneinander. Deren Umsetzung erlangt den Charakter des Erfüllens einer rechtlichen Verpflichtung, die sich am Buchstaben der Formulierung orientiert, nicht aber ein radikaler Gehorsam, der sich mit dem göttlichen Willen identifiziert. Damit stimmen auch solche Vorstellungen überein, wie dass sich anderweitige Fehltritte durch gewisse Taten kompensieren ließen, ebenso wie die von einer Art Heimzahlung (ius talionis), und weiterhin gehört dazu auch die Ungewissheit der Erlösung und das quälende Gefühl der Sündhaftigkeit, mit dem die Selbstgerechtigkeit der Abkehr von den „Sündern und Zöllnern“ kontrastiert, 
sowie schließlich der Umstand, dass die Buße selbst als eine verdienstvolle Tat angesehen wird.

Die Botschaft Jesu ist nun, wie Bultmann schon in seinem ,Jesus“-Buch (1926) aufzeigte, ein großer Protest gegen diese dekadente jüdische Religiosität des Ritualismus, der juristischen Äußerlichkeit und der abergläubischen Bußfertigkeit, ein Protest im Rahmen der jüdischen Frömmigkeit selbst. Diesen Protest charakterisiert Bultmann als eine Radikalisierung des alt-testamentarischen Glaubens im Sinne des Gehorsams vor Gott: Gott verlangt den ganzen Willen des Menschen, nicht nur die äußerliche Erfüllung von Geboten und das bedingungslos, ohne Erleichterung. Angesichts der göttlichen Forderung in ihrer Gesamtheit gibt es keinerlei Freiheit. Der Lohn ist bar jeder Anmessung an die Verdienste. Die Aktualität des alt-testamentarischen Prophetentums erneuernd unterscheidet Jesus kritisch zwischen wesentlichen und nebensächlichen Geboten des Alten Testaments. Nicht die wörtliche Erfüllung, sondern der Geist der Liebe gibt ihnen Sinn und Wert. Die Forderung der Liebe wendet sich an den Einzelnen, doch von großem Gewicht sind ihre sozialen Konsequenzen. Sie ist nicht von vornherein ein Plan zur Reform der Gesellschaft und der Welt, keine Sozialphilosophie, noch stützt sie sich auf dergleichen. Die Forderung ist gegenwärtig, absolut dringlich und in diesem Sinne eschatologisch. Es ist das aber keine Eschatologie der Weltflucht und der Askese, sondern ein unaufhörlicher Verweis auf die direkte Begegnung mit dem Nächsten und eine Hinwendung zur konkreten Lebenssituation. Ihre Botschaft ist vor allem ein Aufruf zur Buße; in ihr wird die Allmacht Gottes anders als in der abstrakten jüdischen Transzendenz wieder zu einer aktuellen, gegenwärtigen Kraft. Gottes Gebot, seine Forderung erlangt dadurch einen neuen Sinn, ebenso aber auch seine Gnade und die Buße - eine Buße, die eng mit der Forderung der Liebe verknüpft ist: zu vergeben, so wie auch wir Vergebung wünschen. Aus dem späten Judentum dringt aber die Enthistorisierung, die Loslösung von der kollektiven Volksgebundenheit der alttestamentarischen Hoffnung, in die Botschaft Jesu ein. Während allerdings die göttliche Transzendenz im Judentum eine Abstraktion des Gemeinwesens ist und die menschliche Transzendenz durch den rituellen Gehorsam gegenüber dem Gesetz gebildet wird, ist Gott bei Jesus transzendent im Sinne des Zukünftigen, des kommenden Herrn, der ständig vor uns steht und durch dessen Forderung die weltliche Verankerung des Menschen bis in ihre Grundfesten erschüttert wird.

Das Hoffen Jesu gehört in den Rahmen der jüdischen Apokalyptik, welche selbst stark aus dem Osten beeinflusst ist, besonders aus dem Iranischen: der Dualismus zweier Äonen. Anzeichen für die Erfüllung der Zeit ist sein eigenes Kommen, auch seine Taten nicht aber in einer objektiv mythischen Bedeutung, sondern nur für den Blick des verinnerlichten Glaubens. Die Austreibung von Dämonen als Wundertat ist natürlich zeitbedingt; 
dahinter steht aber der Aufruf zur Entscheidung angesichts der offenbaren Ankunft des Reiches Gottes: Jesus selbst ist Zeichen und Zeugnis dafür. Der Aufruf zur radikalen Entweltlichung ist hier etwas anderes als Askese: Aufruf, vorbereitet zu sein für das absolute Gebot Gottes. Die Erwartung eines nahen Endes der alten Welt ist keineswegs Voraussetzung, sondern Folge und Indiz für die übergeordnete Bedeutung der Botschaft Jesu: der Anspruch Gottes an den Menschen ist absolut, und deshalb muss der Mensch sich entscheiden. Aus seiner Botschaft entsteht dann dadurch, dass Jesus selbst als der erwartete „Mensch“, als eschatologischer Messias in ihre inhaltliche Bedeutung einbezogen wird (was er nach Bultmann selbst nicht tat), das Christentum. Während also die jüdische Diaspora durch die Übernahme der griechischen Ideen von Kosmos und Paideia die Grundlagen einer Theologie auf metaphysischer Basis vorbereitet, vollzieht sich auf dem Boden Judas selbst die radikalste verinnerlichende Bereinigung des alt-testamentarischen Erbes mit unabsehbaren geschichtlichen Konsequenzen.

Der Überzeugung von der menschlichen Ohnmacht, die im Alten Testament außerordentlich stark blieb, stellt Bultmann den hellenischen Geist im Sinne der Entwicklung menschlicher Überlegenheit gegenüber. Dies natürlich nicht im modernen Sinne praktischer Herrschaft über die Natur, sondern in der rein griechischen Überlegenheit des Geistes über die restliche Physis. So deutet er schon die homerische Religion als eine des Geistes, noch mehr aber die Religiosität der griechischen Polis und das religiöse Fundament der politischen Institutionen. Das Gebot, die Grenze des Menschlichen nicht zu überschreiten, ist auch und vor allem ein Gebot der Selbsterhaltung der Polis, die von jederlei eigenmächtigem Individualismus bedroht wird. Deshalb ist auch die griechische Aufklärung für die religiösen Vorstellungen ebenso ruinös wie für die traditionelle Gesellschaftsordnung. Die Aufklärung setzt an die Stelle göttlicher Autoritäten die Autorität der Wissenschaft, der Lehre von der Physis. Sokrates versucht, die Gemeinschaft mit denselben Mitteln wiederherzustellen, mit denen die übrigen Aufklärer ihren Zerfall bewirken. Bultmann bewegt sich hier im Rahmen traditioneller Ansichten, die von der neueren philosophischen Geschichtsschreibung ziemlich erschüttert wurden: Die ursprüngliche Philosophie ist eine primitive Protophysik, Sokrates lenkt ihre Aufmerksamkeit weg vom Himmel auf die Erde, er vollzieht dies mit Hilfe des Logos als einer Möglichkeit, die Normen menschlichen Handelns zu definieren. Von dort nimmt dann der platonische Idealismus mit dem zentralen Problem des Aufbaus der Polis seinen Ausgangspunkt. Der Logos ist gestützt auf die Schau der ewigen Ideen - dieses geistige Reich ist Platons neue Religion. Die Transzendenz jedoch, von der Platon spricht, ist eine von Grund auf andere als die transzendente Tradition des Alten Testaments. Die Transzendenz der Idee, könnte man sagen, bedingt die Metaphysik der Idee und deren moralisch-politische Ableitung: den vollkommenen Staat. In einer 
schönen, idealtypischen Skizze schildert Bultmann die Grundlagen der griechischen Weltanschauung: ihren Objektivismus und Rationalismus, die rationale Ethik und das Ideal der Bildung, ihr Konzept der Arbeit an sich selbst, die systematische Ethik, ihren mangelnden Sinn für die konkrete, reale Individualität und für die Geschichte - in exakter Opposition zu dem, was oben von der alt-testamentarischen Welt ausgeführt wurde.

Das Christentum aber traf schon gar nicht mehr auf diese klassische griechische Welt - sie war tot, und ihr Erbe überlebte in Form des Hellenismus. Von den hellenistischen Positionen untersucht Bultmann vor allem den Stoizismus. Die Stoa versucht, den klassischen Dualismus wieder in eine Einheit zu überführen, und bringt damit den inneren Konflikt und Zwiespalt des stoischen Weisen hervor. Die stoische Freiheit begreift er als Parallele zur christlichen paulinischen Freiheit: während diese allerdings bei Paulus in der Anerkenntnis der Sündhaftigkeit und der Verantwortlichkeit vor Gott besteht, ist die stoische Freiheit eine vollständige Dehistorisierung, das gänzliche Verlassen allen menschlichen Geschehens, der Rückzug auf den ewigen Kern der Weltsubstanz. Das Problem der Befreiung vom geschichtlichen Fatum, das sich also schon im Stoizismus zeigt, steigert sich weiter in den astrologischen Überzeugungen und Fatalismen des spätrömischen Hellenismus. Ebenso wie das Christentum könnte man Gnosis und Mysterienreligion deshalb als verschiedene Antworten auf dieselbe Frage einer Befreiung vom weltlichen Fatum verstehen. Stoa, Mysterien, Gnosis stellen Etappen einer fortschreitenden Entfremdung des Menschen von der Welt bis hin zum radikalen Dualismus dar. Die Gnosis ist ein vorchristliches Gebilde, eine dualistische Heilsreligion, in ihr zeigt sich zum ersten Mal (im Unterschied zur griechischen Seelenauffassung) die Idee einer radikalen Verschiedenheit der Seele von allem Weltlichen - jedoch ohne die christliche Konkretion und Positivität (d.h. im Grunde ohne Historizität, Zeitlichkeit).

In dieser Hinsicht erweist sich das ursprüngliche Christentum als eine synkretistische Religion, wozu natürlich vor allem die Frömmigkeit und der Kult Jesu beigetragen haben, aber auch die hellenistische Philosophie und Religion, die Dämonologie und die Gnosis. Gleichwohl jedoch - und das ist eigentlich Bultmanns These - zeigt sich am Grunde dieses scheinbaren Konglomerats eine einheitliche, eine neue und originäre Auffassung vom Sinn des Menschlichen - insbesondere eine neue Beziehung zur Zeit, ein neues Weltverhältnis und eine neue Vorstellung der Erlösung. Die radikalste Frage, die sich der antike Mensch stellte, erfährt eine grundsätzliche Veränderung und begründet so die neue Auffassung der Zeit. Auch wenn Sokrates in seinem Wissen des Nicht-Wissens aufweist, dass der Mensch nicht weiß, was - positiv und thematisch - das letzte und umfassende Gute ist, so setzt er doch immer voraus, dass der menschliche Wille wesentlich ein Wollen des Guten ist. Für den hellenischen Menschen ist das Wollen synonym damit, das Gute zu wollen, und 
die Gleichung zwischen beiden bildet das analytische Urteil. Der christliche Mensch hingegen weiß von den wesenhaften Disharmonien des Willens, er weiß, dass es möglich ist, das Gute zu hassen, und dass man sich keineswegs auf ein inneres Erfassen dessen, was richtig und gut ist, verlassen kann - der Mensch verfügt einfach nicht darüber, niemals und auch nicht auf dem sokratischen Umweg über die docta ignorantia, über die Enthüllung und Erniedrigung dessen, der mit seinem vermeintlichen Wissen von Gut und Böse prahlt. Ohnmächtig vor der Frage von Gut und Böse stehend ist der Mensch deshalb immer dem Verfall preisgegeben. Und so wie beim stoischen Weisen besteht auch für den christlichen Menschen die Hauptaufgabe in der Befreiung von der Bürde, die schon immer auf dem Menschen lastet, in der Befreiung von der Verflechtung in die Schicksale dieser Welt. Nur ist es so, dass der Stoiker dieses Problem löst durch eine Entweltlichung, durch Leugnung der Zeit und Verewigung, wohingegen der christliche Mensch sich auf die Zukunft stützt, das Leben in die Zukunft öffnet: Denn nicht in dem, was schon ist, was gegeben ist, sondern in dem, was kommt und uns noch bevorsteht, liegt die eigentliche Erfüllung des Lebens, die es uns möglich macht, auch den schwierigsten Lebenssituationen zu begegnen und sich mit ihnen abzufinden, ja sogar gerade allem Schmerzhaften und Schwierigen eine positive Seite zu entlocken - denn hilft uns dieses Kommende etwa nicht, all das zu überwinden, was schlicht gegeben, fertig, weltlich und vergangen ist? In seiner Annahme der Botschaft dessen, was immer künftig ist, überwindet der christliche Mensch das Gewicht der Welt wie auch seine eigene weltliche Schwere, er transzendiert, aber in der Welt: er fällt nicht in die Ewigkeit des Geistes wie der Platoniker, nicht in die Ewigkeit der Weltsubstanz wie der Stoiker, nicht in eine Ewigkeit des Abtauchens in die überweltliche Unversehrtheit wie der Gnostiker oder Neuplatoniker - sondern er fällt in diese Welt, die in jedem Augenblick, in jeder Situation von der absoluten Kraft der göttlichen Forderung angehoben und durchleuchtet wird, in diese konkrete Welt, die er umgestalten soll in die neue, brüderliche Gemeinschaft vor dem Angesicht Gottes.

Mit der Entdeckung dieser konkreten historischen Zeit, einer ewig reinen Zukunft, unbelastet von jeder Bindung an das, was schon ist, kann der christliche Mensch dem Kampf mit der Welt einen anderen Sinn geben als die Stoiker und die Gnostiker. Der Kampf mit der Welt ist zwar ebenso wie in der Gnosis ein Kampf mit dämonischen Mächten, aber viel tiefer ein Kampf mit den begrenzenden und in die Vergangenheit hineinbannenden „Kräften“ der Welt, wie Sinnlichkeit, Gesetzmäßigkeit, Sünde und Tod. Aber es ist dies kein Kampf um die Wiedergewinnung des seelischen Funkens hin zu einer uranfänglichen Reinheit, sondern ein Kampf, der in die Zukunft blickt, auf die Gnade Gottes. Und diese Gnade Gottes ist eben die Transzendenz Gottes: also keineswegs bloß irgendein ob- 
jektives Bild, und sei es auch noch so großartig, sondern ein Aufruf dessen, was nie vergangen sein und so auch nie überwunden werden kann.

Göttliche Gnade und Erlösung werden zwar auch mit Hilfe eines traditionellen Gerüsts gedacht, das einesteils aus dem jüdischen Messianismus ererbt ist, andernteils und hauptsächlich aus der Gnosis mit ihrer Vorstellung vom Kommen des Erlösers, der einen neuen kosmischen Äon begründen wird. Aber dieses Gerüst dient allein dazu, die Gegenwart des Heilsgeschehens, die Gegenwart der Glaubensentscheidung zu betonen. (Mir scheint an dieser Stelle unzweideutig aufzuleuchten, dass Bultmann die kosmische Funktion Jesu für ein Moment der zeitgenössischen Mythologie hält, ohne welches das Hören der Botschaft und ihre menschliche Rezeption nicht auskäme; die Göttlichkeit Jesu muss dann also auch einen anderen Sinn als den traditionellen, mythisch-metaphysischen haben.) Diese Gegenwart des Heils zeigt sich nicht in irgendwelchen sinnlich-objektiven, kosmologischen Andeutungen, sondern durch einen insgesamt veränderten Weltbezug, der nicht bloße Negation ist, wie in den asketisch-mythischen Religionen, sondern Distanz - aber eine, bei der die konkrete Arbeit, das Tätigsein und Handeln, die Liebe, die Anstrengung, die Praxis wesentlich werden, nicht nebensächlich bleiben und unverbunden vor der Ewigkeit stehen. Die Welt verschwindet nicht, noch flieht der Mensch aus ihr - aber sie wird leicht.

Oft wird gegen Bultmann eingewandt, seine Lehre sei eher anthropologisch als theologisch. Ich meine, dass es eine echte Theologie ist, die allerdings indirekt über Gott spricht, indem sie nämlich ausdrückt, auf welche Art und Weise der Glaube den Menschen öffnet. Er gelangt so für das Christentum zu Formulierungen, die durch Einfachheit und Tiefe überraschen.

Vor einhundertfünfzig Jahren stellte ein großer deutscher Philosoph auf ziemlich ähnliche Weise die Frage nach der Beziehung zwischen Altem Testament, Hellenismus und Christentum: auf ähnliche Weise verfolgte er auch die Antithese von Judentum und griechischer Polis, in den Details sicher nicht so historisch gelehrt wie Bultmann, aber mit einer gewaltigen Kraft der Anschauung. Das Judentum galt ihm als die Religion menschlicher Unfreiheit, das Griechentum schien sich ihm auf eine Religion des Geistes und der Schönheit zu stützen, es war ihm eine Welt der Harmonie und der Freiheit des Menschen. Auch zwischen Stoizismus und Christentum sah er einen Zusammenhang, ebenso wie Bultmann eine Parallele zwischen der Freiheit des stoischen Weisen und des paulinischen Christen erkennt. Aber während sich Hegel von Anfang an für den griechischen Maßstab entschied den Standpunkt der menschlichen Vorherrschaft über die Welt und in der Welt, was ihn mit Umwegen über das christliche Mittelalter, die Welt der Geknechteten, und über den Aufstand dieser Unterjochten zur Realisierung des verallgemeinerten griechischen Ideals einer herrschaftlichen politischen Freiheit führt - verfolgt Bultmann die Idee des Vorrangs des 
alt-testamentarischen Maßstabs, des Maßstabs der menschlichen Abhängigkeit und seiner Unfähigkeit, aus eigenen Kräften zu einer Beherrschung und Sinngebung des gesamten Universums zu gelangen. Auch hier rückt der Begriff der Geschichtlichkeit ins Zentrum, doch es wird daraus keine Metaphysik der Geschichte. Verständlich wird vielmehr das geschichtliche Wirken des Christentums wie auch all der Ideologien, die auf seiner Grundlage entstehen. Und es erhebt sich daraus der wiederholte Aufruf an die Philosophie, die Konsequenzen zu ziehen aus dem Problem, das sich zwischen den von Hegels Phänomenologie des Geistes und Bultmanns Urchristentum bezeichneten Polen aufgetan hat, und dieses einer Lösung näherzubringen.

Übersetzung aus dem Tschechischen: Ludger Hagedorn 